\title{
Observations of pulsars at 9 millimetres
}

\author{
O. Löhmer ${ }^{1}$, A. Jessner ${ }^{1}$, M. Kramer ${ }^{2}$, R. Wielebinski ${ }^{1}$, and O. Maron ${ }^{3,1}$ \\ 1 Max-Planck-Institut für Radioastronomie, Auf dem Hügel 69, 53121 Bonn, Germany \\ e-mail: jessner@mpifr-bonn.mpg.de \\ 2 University of Manchester, Jodrell Bank Centre for Astrophysics, Manchester M13 9PL, UK \\ 3 J. Kepler Institute of Astronomy, University of Zielona Gora, Poland
}

Received 24 November 2006 / Accepted 11 December 2007

\section{ABSTRACT}

\begin{abstract}
Aims. The behaviour of the pulsar spectrum at high radio frequencies can provide decisive information about the nature of the radio emission mechanism.

Methods. We report recent observations of a selected sample of pulsars at $\lambda=9 \mathrm{~mm}(32 \mathrm{GHz})$ with the 100 -m Effelsberg radio telescope.

Results. Three pulsars, PSR B0144+59, PSR B0823+26, and PSR B2022+50, were detected for the first time at this frequency. We confirm the earlier flux density measurements for a sample of six pulsars, and we are able to place upper flux density limits for another 12 pulsars. We find that all pulsar spectra have a simple form that can be described using only three parameters, one of which is the lifetime of short nano-pulses in the emission region. The study of the transition region from coherent to incoherent emission needs further and more sensitive observations at even higher radio frequencies.
\end{abstract}

Key words. stars: pulsars: individual: PSR B0144+59 - stars: pulsars: individual: PSR B0823+26 -

stars: pulsars: individual: - PSR B2022+50 - radiation mechanisms: non-thermal - radio continuum: stars

\section{Introduction}

Almost 40 years after the discovery of pulsars, it is still not possible to give a detailed explanation of the radio emission process, and a large number of fundamental questions remain (e.g. Lorimer \& Kramer 2005) to be answered. Advances in our theoretical understanding of the radio emission and the associated conditions in the pulsar magnetosphere (e.g. Petrova 2001; Melrose et al. 2006) are still largely driven by observational progress (e.g. Kramer et al. 2006).

In recent years, most observational studies pertaining to emission processes have concentrated on polarisation properties (e.g. Karastergiou \& Johnston 2004) or on the drift behaviour of sub-pulses (e.g. Weltevrede et al. 2006). However, as past studies of other discrete radio sources have demonstrated, the investigation of radio frequency spectra can be crucial for identifying of the underlying emission process (e.g. Conway et al. 1963). Although we may expect that similar detailed studies of pulsar spectra can be helpful in solving the "pulsar problem", such studies (e.g. Malofeev et al. 1994) have so far not been successful in establishing a relationship between pulsar spectra and other observed properties. It turned out that significant correlations between spectral properties and pulsar spin parameters could not be found or did not survive more detailed scrutiny (Maron et al. 2000). It is therefore more likely that spectral studies of separate profile components (Kramer 1994) or even of individual pulses (Kramer et al. 2003; Karastergiou \& Johnston 2006) are better suited to addressing questions about the emission process, as the average pulse profile seen at different frequencies is dominated by different components with different spectral behaviours. Differences in the flux density spectra of separate components can indeed explain certain features in observed spectra of the average profiles, such as breaks in power laws, but may also contribute to the observed polarisation evolution with frequency (Karastergiou et al. 2005). Other authors have, however, claimed that the general decrease in polarised emission at high frequencies is due to a frequencydependent decrease in the coherence of pulsar radio emission (e.g. Xilouris et al. 1996).

The steepness of pulsar spectra (mean spectral index of $\alpha=-1.6$ with indices between $\alpha \sim 0$ and $\alpha \sim-3$ for power law spectra, $S \propto v^{\alpha}$, Maron et al. 2000) may be caused by the mechanisms that enforce the coherence of pulsar radiation as has been suggested by Michel (1991). This concept is based on a model in which an initially incoherent radiation process produces a coherent output by means of antenna mechanisms or resonances. The radiation produced by other considered processes, especially maser mechanisms and plasma radiation, is however inherently coherent. Should the first class of models be applicable, then pulsar radiation would be a combination of coherent and incoherent emission, where the coherent emission is seen to dominate at radio frequencies, establishing a typical coherence length $\lambda_{\mathrm{c}}$ beyond which incoherent emission should dominate. Interestingly, the non-thermal emission of pulsars observed at infrared and optical frequencies is indeed believed to be due to incoherent synchrotron emission (Crusius-Waetzel \& Lesch 2002).

The study of pulsars at very high radio frequencies, where the coherent emission process might become less dominant, is an obvious procedure for investigating a possible intrinsic coherence length and the contribution of an additional incoherent emission component. So far, the flux density spectrum of pulsar emission in the transition region between the coherent radio emission and the incoherent infrared emission is unknown. We therefore aim to investigate the exact position and nature of this transition from coherent to incoherent emission through access 
to the spectral region where the spectral index is flattening or perhaps showing an upturn.

The information gap between high radio and infrared frequencies was first pointed out after early observations of the Crab pulsar (e.g. Smith 1977) at radio and optical wavelengths. The Crab pulsar, among a few others, also provides direct evidence about the fine details of the radio-emission process in the form of giant radio pulses (GRPs). These have been observed up to $15 \mathrm{GHz}$ for the Crab pulsar (Hankins private communication, Kondratiev et al., in preparation) and occur during all rotation phases where the average pulse profile shows radio emission (Jessner et al. 2005). It is worthwhile investigating the possibility of the spectrum, or parts of it, being the natural result of a superposition of a very large number of very short pulses, similar to the "nano-shots" (Eilek \& Hankins 2006) observed for the Crab pulsar. One can show that a simple form of the spectrum will be the result, depending - apart from a scale factor - on at most two more parameters, one of which is the typical decay time of the nano-shots.

The filling of the spectral gap, hence the attempt to learn more about the radio emission process by studying this crucial frequency range, was one of the motivations for the technically challenging observations of pulsars at mm-wavelengths (Wielebinski et al. 1993; Kramer et al. 1996). Only at high frequencies will propagation effects become negligible for the majority of pulsars, their intrinsic emission properties may reveal themselves only here. This is commonly appreciated for the interstellar medium, but it may even be important for propagation effects in the pulsar magnetosphere (e.g. Hoensbroech \& Lesch 1999).

As we summarise in the following, the first observations of pulsars at mm-wavelengths provided some unexpected results, which triggered further follow-up studies. The results of such a study are described in this paper, confirming the results for the known detected pulsars but with an additional enlargement of the numbers of observed pulsars to provide better statistics.

In this paper we briefly review the main properties of pulsar spectra, before we describe new observations of pulsars at mmwavelengths and present their results. We then compare these new results with previous measurements before we discuss the implications of our results and our conclusions are drawn.

\section{Pulsar spectra}

Prior to the observations by Wielebinski et al. (1993), it was commonly believed that the generally steep pulsar spectrum continues to the highest radio frequencies (e.g. Manchester \& Taylor 1977; Malofeev et al. 1994). On the other hand, one can even find claims in the literature that pulsar spectra show a "cutoff" at about $10 \mathrm{GHz}$ or so (e.g. Gurevich et al. 1993). This erroneous impression was caused by the lack of large sensitive telescopes and high-frequency receivers at that time. The absence of any cut-off was demonstrated by the first detection of four pulsars at $33.8 \mathrm{GHz}(\lambda=8.9 \mathrm{~mm}$ Wielebinski et al. 1993). In fact, two pulsars were stronger at these very high radio frequencies than was previously thought. In contrast to predictions based on the extrapolation of the spectra measured up to $\sim 24 \mathrm{GHz}$, the measured flux densities of these two sources (PSRs B1929+10 and B2021+51) were factors of five to ten higher than expected. This apparent spectral turn-up was further investigated by Kramer et al. (1996). With their observations between $\sim 27 \mathrm{GHz}$ and $\sim 35 \mathrm{GHz}$, they confirmed the results for B1929+10 and B2021+51, but also established a normal continuation of the known spectrum for six additional sources. The four pulsars strongest at $32 \mathrm{GHz}$ were later also successfully observed at $43 \mathrm{GHz}(\lambda \sim 7 \mathrm{~mm})$, underlining the impression that pulsar spectra show unusual behaviour at high radio frequencies; i.e. they may become flatter or even possibly show a turnup at mm-wavelengths (Kramer et al. 1997). The first and so far only detection ${ }^{1}$ of a radio pulsar at $87 \mathrm{GHz}(\lambda=3 \mathrm{~mm})$ with the 30-m Pico Veleta telescope (Morris et al. 1997) shows a continuation of the relatively flat spectrum of the detected pulsar B0355+54. However, recent reports suggest that turn-ups in pulsar spectra may even occur at much lower radio frequencies (Kijak \& Maron 2004). Clearly, more studies of more pulsars at $\mathrm{mm}$-wavelengths are required.

\section{Observations}

We selected a sample of 15 pulsars from the catalogue of Maron et al. (2000), from the list of brightest pulsars, and from the list of pulsars with unusual frequency evolution given by von Hoensbroech \& Lesch (1999). All observations were made with the 100-m Effelsberg radio telescope of the Max-PlanckInstitut für Radioastronomie in Bonn, Germany. We observed the sources at three separate epochs with good to excellent weather conditions: 2002 March, 2002 September, and 2003 January. Integration times of the pulsars were between 30 and $180 \mathrm{~min}$, depending on the expected flux density and visibility of the sources.

We used an HEMT receiver installed in the secondary focus with a fixed central frequency of $32 \mathrm{GHz}(\lambda=9.37 \mathrm{~mm})$. The total bandwidth of the system was $2 \mathrm{GHz}$, with a receiver noise temperature of about $60 \mathrm{~K}$. The telescope gain at this frequency is $\sim 1 \mathrm{~K} / \mathrm{Jy}$. The receiver provided LHC and RHC signals that were digitised and independently sampled every $P / 1024 \mathrm{~s}$ and synchronously folded with the topocentric pulse period $P$, using the Effelsberg Pulsar Observation System (EPOS, Jessner 1996). Records of 15 s sub-integrations were transferred to disk for offline data analysis.

For flux-calibration purposes, we made use of an internal noise diode fed directly into the waveguide following the feed horn. EPOS allows us to switch this noise diode synchronously with the pulse period, occupying the first 50 phase bins of the pulse period in the measured profile. To check that the actual pulsar signal, expected to be relatively weak at $32 \mathrm{GHz}$, does not overlap with the strong, artificial calibration signal, we also observed each pulsar briefly at $4.85 \mathrm{GHz}$ and $8.35 \mathrm{GHz}$, making sure that it is positioned correctly, and then switched to the high frequency receiver in less than $30 \mathrm{~s}$ that way preserving the pulse phase for all frequencies. The strong and clearly visible pulsar signals observed at the two lower frequencies could then be directly related to the possible signal of the high frequency emission, greatly facilitating confirmation of a possible detection.

Flux densities were obtained for all three frequencies by comparing the pulse profiles to the corresponding signal of the noise diode, which itself was calibrated by observing known reference sources during regular pointing observations. The continuum references (NGC 7027, 3C 286, and NRAO 150) were

\footnotetext{
1 Here we are not counting the recent detection of radio emission at $144 \mathrm{GHz}$ (Camilo et al. 2007) from the magnetar XTE J1809-194 as typical pulsar emission. Although it is accepted that these sources are rotating and magnetised neutron stars, their variability and spectra differ significantly from ordinary pulsars (Kramer et al. 2007; Lazaridis et al., in preparation; Stappers et al., in preparation). An implicit assumption that their emission process is the same as that of ordinary pulsars cannot be justified at present.
} 
Table 1. Results of observations at $32 \mathrm{GHz}$.

\begin{tabular}{|c|c|c|c|c|}
\hline PSR & $\begin{array}{l}\text { Pulses } \\
\text { total }\end{array}$ & $\begin{array}{l}\text { Time } \\
(\min )\end{array}$ & $\begin{array}{r}\text { Flux density } \\
(\mathrm{mJy})\end{array}$ & $\begin{array}{l}\text { No. of } \\
\text { measurements }\end{array}$ \\
\hline \multicolumn{5}{|c|}{ New detections: } \\
\hline B $0144+59$ & 30552 & 99.8 & $0.062 \pm 0.006$ & 2 \\
\hline \multirow[t]{2}{*}{ B $0823+26$} & 24752 & 218.9 & $0.023 \pm 0.0010$ & 2 \\
\hline & 12516 & 111.0 & $<0.170$ & $0(\mathrm{~K} 95)^{*}$ \\
\hline \multirow[t]{2}{*}{ B $2022+50$} & 9720 & 60.4 & $0.046 \pm 0.009$ & 1 \\
\hline & \multicolumn{4}{|c|}{ Re-detections: } \\
\hline \multirow[t]{2}{*}{ B $0355+54$} & 17480 & 45.6 & $0.76 \pm 0.14$ & 2 \\
\hline & 57095 & 148.8 & $0.8 \pm 0.2$ & $6(\mathrm{~K} 95)$ \\
\hline \multirow[t]{2}{*}{ B1133+16 } & 2148 & 42.5 & $0.055 \pm 0.06$ & 1 \\
\hline & 13920 & 275.4 & $0.03 \pm 0.02$ & $2(\mathrm{~K} 95)$ \\
\hline \multirow[t]{2}{*}{ B1706-16 } & 8712 & 94.8 & $0.07 \pm 0.01$ & 1 \\
\hline & 11154 & 121.2 & $0.06 \pm 0.01$ & 2 (K95) \\
\hline \multirow[t]{2}{*}{ B1929+10 } & 15708 & 59.3 & $0.19 \pm 0.02$ & 1 \\
\hline & 133188 & 502.8 & $0.21 \pm 0.01$ & 6 (K95) \\
\hline \multirow{2}{*}{ B $2020+28$} & 6278 & 35.9 & $0.06 \pm 0.01$ & 1 \\
\hline & 41065 & 235.2 & $0.09 \pm 0.02$ & 1 (K95) \\
\hline \multirow{2}{*}{ В $2021+51$} & 3752 & 33.1 & $0.28 \pm 0.03$ & 1 \\
\hline & 61096 & 349.8 & $0.323 \pm 0.007$ & 9 (K95) \\
\hline \multicolumn{5}{|c|}{ Upper flux limits: } \\
\hline B $0154+61$ & 4578 & 179.0 & $<0.06$ & \\
\hline $\mathrm{B} 0611+22$ & 32076 & 179.0 & $<0.09$ & \\
\hline B0628-28 & 2256 & 46.8 & $<0.3$ & \\
\hline B0740-28 & 16554 & 46.0 & $<0.17$ & \\
\hline B1604-00 & 5460 & 38.4 & $<0.13$ & \\
\hline B1642-03 & 7334 & 47.4 & $<0.3$ & \\
\hline B1822-09 & 2888 & 37.0 & $<0.13$ & \\
\hline $\mathrm{B} 1935+25$ & 6734 & 22.6 & $<0.9$ & \\
\hline $\mathrm{B} 2000+32$ & 8946 & 103.9 & $<0.08$ & \\
\hline В2319+60 & 3168 & 119.0 & $<0.3$ & \\
\hline $\mathrm{B} 2323+63$ & 2490 & 59.6 & $<0.6$ & \\
\hline B2334+61 & 4830 & 39.9 & $<0.4$ & \\
\hline
\end{tabular}

* The designation (K95) refers to earlier observations reported by Kramer (1995).

observed approximately every hour, checking both pointing and focus stability. Typical pointing errors were rms $\sim 5^{\prime \prime}$, compared to a beamwidth of $23^{\prime \prime}$ at $32 \mathrm{GHz}$. Flux densities for the chosen reference sources were obtained from the catalogue of Peng et al. (2000). Uncertainties in the resulting pulsar flux densities are estimated to be about $20 \%$ for a single observation.

\section{Results}

In total, we observed a sample of 21 pulsars. The results of the measurements are summarised in Table 1 . We were able to detect PSRs B0144+59, B0823+26, and B2022+50 at $32 \mathrm{GHz}$ for the first time, determining their flux densities at their so far highest observation frequency. The time-aligned profiles of these sources measured at 4.85, 8.35, and $32 \mathrm{GHz}$ are shown in Fig. 1, while their resulting spectra are displayed in Fig. 2. Flux densities shown for lower frequencies are taken from the literature (Maron et al. 2000) or from unpublished observations.

We also successfully observed all those pulsars that were already detected by Wielebinski et al. (1993), Kramer (1995), and Kramer et al. (1997). The measured flux densities are in very good agreement with previous observations.

For those pulsars that were not detected, we estimate upper flux limits following the procedure described by Sieber, \& Wielebinski (1987). The upper limit is based on an estimate of five times the RMS of the noise signal multiplied by the equivalent pulse width. For our sample we used the pulse width $W_{50}$ at the highest known frequency as given in the EPN pulsar data base $^{2}$ or by Kramer et al. (1994) or Kijak et al. (1998).

\section{Discussion}

\subsection{Turn-up at high frequencies?}

Our results confirm all previously published measurements of flux densities of pulsars at mm-wavelengths. We also present spectral information for three newly detected pulsars at mmwavelengths. No new turn-up in the spectrum was found. The spectra of the newly detected pulsars follow the trends determined from observations at lower radio frequencies. While on one hand this gives us great confidence in the reliability of the adopted calibration procedure, it does suggest, on the other, that a spectral turn-up at a frequency as low as $30 \mathrm{GHz}$ is the exception rather than the rule. We note that the spectrum of PSR B0144+59 shows a peculiar "kink" at $3 \mathrm{GHz}$ to $10 \mathrm{GHz}$, which may be misinterpreted as a "turn-up" or definite "flattening" unless data above $10 \mathrm{GHz}$ are considered (cf. Kijak \& Maron 2004). We believe that this is caused by the peculiar highfrequency profile evolution of this source, which will be studied elsewhere. In any case, it adds to the notion that some pulsar spectra may not be adequately described by simple power laws. Moreover, in view of the evidence that individual pulsar spectra are quite different (differing not only in shape but also e.g. in the location of the low-frequency turn-over, spectral index, and the possible existence of a spectral steepening (Maron et al. 2000), it would be rather naive to assume that all pulsars show identical behaviour in the narrow frequency range probed by our mmobservations. Hence we should not expect to detect a spectral turn-up for all pulsars at $32 \mathrm{GHz}$. Instead, the known diversity of pulsar properties may indeed eventually reveal a turn-up or flattening of pulsar spectra at higher or even at lower frequencies (Kijak \& Maron 2004).

\subsection{The shape of pulsar spectra}

Although pulsar spectra are traditionally described by a power law $S_{v} \propto S^{\alpha}$ with $\alpha$ about -1.6 (Sieber 1973), many of the observed spectra turned out to fit badly to that concept. A number of additional characteristic features are traditionally employed to describe more complex pulsar spectra : some of them exhibit a turn-over at low $v$, some have a "broken power law", some a turn-up or flattening, etc. The original rationale was the analogy of synchrotron radiation, which has a power-law spectrum, so that radio astronomers initially tried to model most observations in terms of power laws. But one of the implicit assumptions was that one wanted to model a temporarily continuous and spatially large-scale radiation mechanism. The observations have, however, come up with strong variability on timescales from days down to nanoseconds (GRPs). A few attempts have been made to fit another function to a pulsar spectrum, most notable Ochelkov \& Usov (1984), who proposed a six-parameter model equation:

$$
S(v)=\frac{S_{0} \cdot v^{a}}{\left(1+\left(\frac{v}{v_{b}}\right)^{b}\right)\left(1+\left(\frac{v}{v_{\mathrm{c}}}\right)^{c}\right)}
$$

Nearly all observed spectra can be described with a suitable choice of these six parameters. In Ochelkov \& Usov's model of curvature radiation of plasma bunches, the parameters $a, b$, and $c$

\footnotetext{
2 http://www.jb.man.ac.uk/research/pulsar/Resources/ epn/
} 


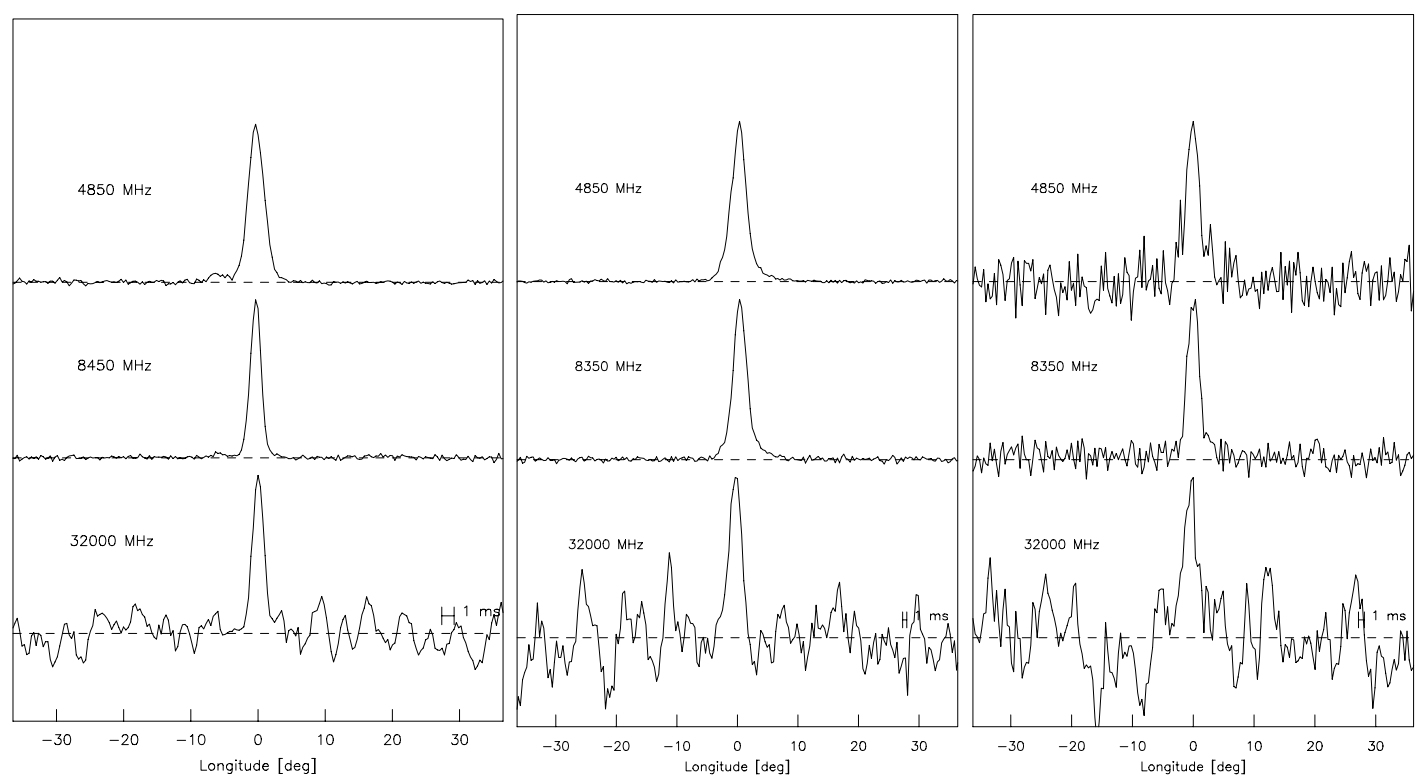

Fig. 1. Time-aligned profiles of the three PSRs B0144+59, B0823+26 and B2022+50 at centre frequencies of 4.85, 8.35 and $32 \mathrm{GHz}$.
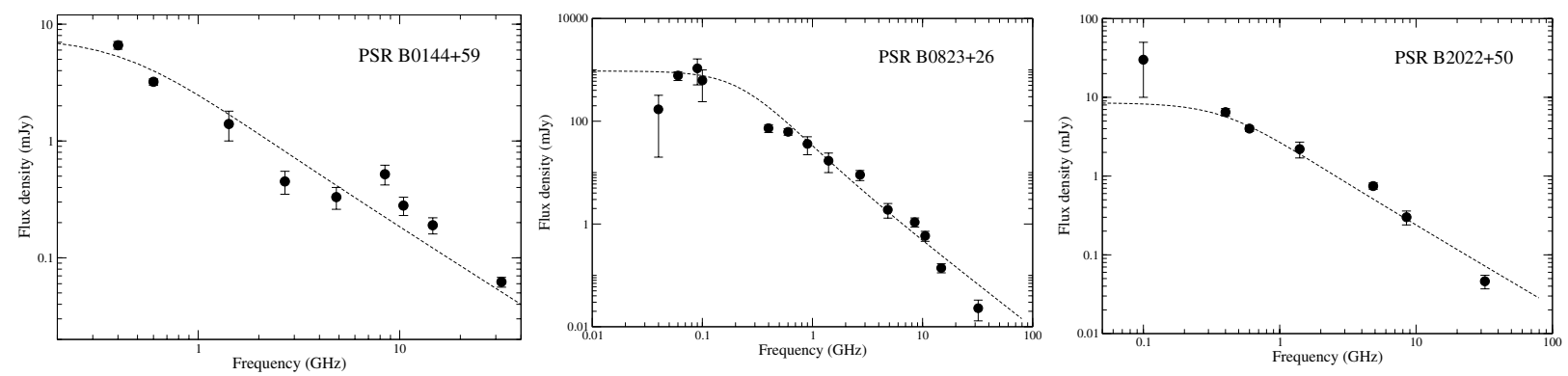

Fig. 2. Resulting flux density spectra for PSRs B0144+59, B0823+26, and B2022+50. Low-frequency data have been taken from available literature (see text). The dashed line shows the model fit with parameters listed in Table 2.

have fixed algebraic relationships, and only two of them should suffice to describe any spectrum. Malofeev et al. (1994) showed, however, that the model parameters were not constrained by the already available data; hence, one may well ask oneself if that is the simplest possible description of a pulsar spectrum.

Do we know of other natural processes, involving flowing charges, that produce spectra of the observed form? Indeed we do, solid-state currents in everyday materials exhibit a nonthermal low frequency noise, often called "flicker noise", "1/fnoise" or simply lf-noise. The spectrum is very similar to a pulsar spectrum, but at frequencies lower by a factor of $10^{7}-10^{8}$. That in itself is not surprising, as the conditions on the pulsar surface and in the magnetosphere are also many orders of magnitude different from our experience with everyday materials. People had already been studying that kind of noise in solids and on surface boundaries in the 1950's (Pfeifer 1959; Bess 1953). It is indeed surprising how far one can get using this very simple one-parameter theory.

We already know from observations that pulsar radio emission varies on very short timescales. Very high-resolution observations of giant radio pulses have so far not found a shortest timescale for the emission, although they have reached nanosecond to sub nanosecond resolution (Hankins et al. 2003; Jessner et al. 2005; Eilek \& Hankins 2006) for the Crab pulsar. Eilek \& Hankins (2006) report an apparent increase in micro-burst duration $\propto v^{-2}$, nano-shots with $\delta t<1 \mathrm{ns,} \mathrm{and} \mathrm{they} \mathrm{infer} \mathrm{that} \mathrm{each}$ giant pulse is a coherent superposition of a number of powerful nano-pulses. Based on these theoretical studies and on the observational evidence, we assume that all pulsar radio emission is a superposition of short-lived $(\tau \sim \mathrm{ns})$ elementary emission processes. The observed shape of the spectrum may then be used to provide constraints on the timescales of the elementary processes.

A shot noise model for the emission due to short-lived nonGaussian elementary processes in ordinary pulsars has been proposed by Jenet et al. (2001). However, in this study we use a different elementary process, one that has been commonly used to model flicker noise in solids and vacuum discharges, and evaluate the expected observable broad band radio spectrum.

Let us assume that we have a stochastic formation of radio emission centres that are characterised by a localised potential $\Phi$ with a lifetime (observer system) of i.e. $\tau_{\mathrm{e}} \sim 10^{-10} \mathrm{~s}$, very similar to the nano pulses proposed i.e. by Weatherall (1998) and also evident in the PIC-simulations of Jaroschek \& Lesch (2006). The decay of the electric potential of a nano-pulse may be described by $\Phi(t)=\Phi_{0} \cdot \mathrm{e}^{-t / \tau_{\mathrm{e}}}$ with $(t>0)$. A random superposition of short pulses has the simple spectrum

$S(\omega)=\frac{S_{0}}{1+\omega^{2} \tau_{\mathrm{e}}^{2}}$

and most of the observed pulsar spectra fit extremely well within a reasonable range of $\tau_{\mathrm{e}}$. The model spectrum depends only the reference flux and a characteristic time $\tau_{\mathrm{e}}$ for the nano-burst decay time. Figure 3 shows the fit to the spectrum of B2021+51, 


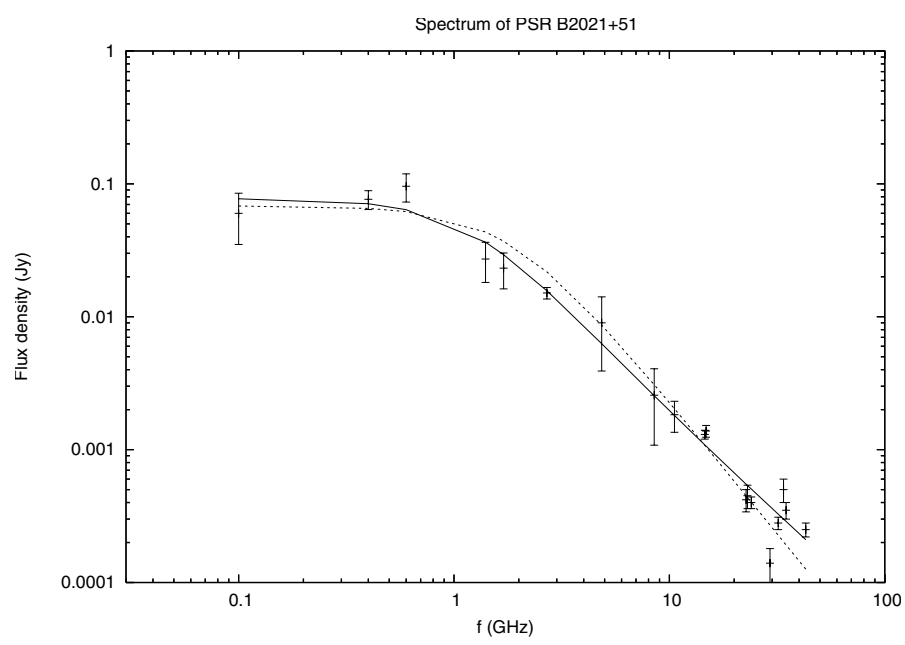

Fig. 3. Spectral fits for PSR B2021+51 using additional data from O. Maron et al. Solid: fit with a spectrum according to Eq. (3) with $S_{0}=1.61 \mathrm{Jy}, \tau_{\mathrm{e}}=0.124 \mathrm{~ns}$, and $n=0.275$. Dashes: Fit with $S_{v}(v)=\frac{S_{0}}{1+(2 \pi v)^{2} \tau_{\mathrm{e}}^{2}}$, here $S_{0}=68 \mathrm{mJy}$ and $\tau_{\mathrm{e}}=0.09 \mathrm{~ns}$.

which extends up to $43 \mathrm{GHz}$. Here we find $\tau_{\mathrm{e}}=0.09 \mathrm{~ns}$. Although the fits using such a simple function are already surprisingly good, they can be improved by extending the analogy to current noise processes (Bess 1953) a bit further. The theory was found to describe noise process currents through solid state boundaries quite well (Pfeifer 1959). Details of the derivation and its connection to a statistical approach to pulsar radio emission processes will be given in a forthcoming paper (Jessner et al., in preparation). Let us assume that these nano-pulses have a spatial cross section of $\sigma_{\mathrm{c}}$ and exist only in small regions of mean radius $r_{0}$ and thickness $\delta$. They are supposed to diffuse through the region on a timescale of $\tau_{0}$. Then again, such a nano-pulse may be described by the evolution of its potential $\Phi(t)=\Phi_{0}\left(\frac{\tau_{0}}{\tau+\tau_{0}}\right)^{n} \cdot \mathrm{e}^{-t / \tau_{\mathrm{e}}}$ with the exponent $n=\frac{\sigma_{\mathrm{c}} \tau_{0} c}{\pi \delta \tau_{0}^{2}}$. The spectrum from a superposition of such pulses can again be found from their Fourier transform and be written in the form

$S(\omega)=S_{0}\left(\frac{1+\omega^{2} \tau_{\mathrm{e}}^{2}}{\tau_{\mathrm{e}}^{2}}\right)^{n-1} \cdot \mathrm{e}^{-\mathrm{i}(n-1) \cdot \operatorname{atan}\left(\omega \tau_{\mathrm{e}}\right)}$.

All observed pulsar radio spectra can easily be fitted using only the three parameters $S_{0}, \tau_{\mathrm{e}}$, and $n$ as seen in in the example of B2021+51 in Fig. 3. We now find a larger $\tau_{\mathrm{e}}=0.124 \mathrm{~ns}$, as Eq. (3) usually improves the fit for the low frequency $(<1 \mathrm{GHz})$ part of the spectrum. However, only the characteristic time $\tau_{\mathrm{e}}$ of the nano-pulses can be directly inferred from the fit to the spectrum, while other physical parameters are being constrained in combination by the value of the exponent $n$. Table 2 lists the results for those pulsars that have been observed up to very high frequencies. The spectra obtained for the pulsars newly detected at $32 \mathrm{GHz}$ are shown in Fig. 2. One notices that the characteristic timescales $\tau_{\mathrm{e}}$ are all found to be in the appropriate range of $0.1 \mathrm{~ns}<\tau_{\mathrm{e}}<2.0 \mathrm{~ns}$. The example of GRP sources like the Crab pulsar provides direct evidence that radio emission processes occur on very similar timescales. The spectra of the above sources can be interpreted as indirect evidence that these are also intrinsic timescales for the emission of ordinary pulsars, where observational constraints do not allow us to resolve the emission on these timescales. But although the Crab pulsar does provide the best direct evidence for nanopulse emission in all its components (Jessner et al. 2005), it
Table 2. Parameters of spectral fits for a number of pulsars.

\begin{tabular}{cccc}
\hline \hline PSR & $\begin{array}{c}S_{0} \\
(\mathrm{Jy})\end{array}$ & $\begin{array}{c}\tau_{\mathrm{e}} \\
(\mathrm{ns})\end{array}$ & $n$ \\
\hline $\mathrm{B} 0144+59$ & 0.03 & 0.34 & 0.454 \\
$\mathrm{~B} 0329+59$ & 22.0 & 0.16 & 0.073 \\
$\mathrm{~B} 0355+54$ & 0.27 & 0.27 & 0.498 \\
$\mathrm{~B} 0628-28$ & 3.09 & 0.62 & 0.114 \\
$\mathrm{~B} 0823+26$ & 1.77 & 0.71 & 0.167 \\
$\mathrm{~B} 0950+08$ & 7.68 & 0.42 & 0.115 \\
$\mathrm{~B} 1133+16$ & 4.41 & 0.51 & 0.103 \\
$\mathrm{~B} 1706-16$ & 0.40 & 0.91 & 0.272 \\
$\mathrm{~B} 1929+10$ & 2.59 & 0.57 & 0.233 \\
$\mathrm{~B} 2021+51$ & 1.61 & 0.12 & 0.275 \\
$\mathrm{~B} 2020+28$ & 1.54 & 0.24 & 0.196 \\
$\mathrm{~B} 2022+50$ & 0.03 & 1.77 & 0.479 \\
\hline
\end{tabular}

cannot serve as a good example for the indirect evidence through its spectrum. The individual components show very distinct evolution with frequency and different nano-pulse characteristics (Eilek \& Hankins 2006), whereas the radio fluxes are usually averages over all components. Furthermore, the duration of the bursts of nano-shots scales as $v^{-2}$, which will add another complication to attempts to model the radio spectrum of that unique source.

\section{Conclusions}

We have presented the first detection of three more pulsars at mm-wavelengths, while presenting upper limits of flux densities for 12 other pulsars. None of these upper limits is yet low enough to establish the nature of the flux density spectrum of those sources, the continuation of the previous spectral trends, or a possible flattening or turn-up at high frequencies. Indeed, while the three newly detected pulsars show a continuation of the lower frequency spectra, we confirmed the flux densities of six previously detected pulsars. This sample of re-detected pulsars includes PSRs B1929+10 and B2021+51, thus giving further credibility to their published flux density measurements, which led to the suggestion of a spectral turn-up at mm-wavelengths for these pulsars. Establishing such a trend could provide a possible link between the spectrum of the coherent radio emission and the incoherent higher frequency radiation, but more sensitive observations at even higher frequencies are needed to substantiate any conclusion in this direction.

We have also found evidence that a heuristic model of pulsar radio emission, consisting of a superposition of a large number of short pulses of only nano-second duration describes observed pulsar radio spectra quite naturally. Apart from intensity scaling, a model fit needs only two parameters, one being the typical nano pulse life time $\tau_{\mathrm{e}}$, the other $(n)$ related to the geometry of the individual emission process. These processes already play a role, with much greater magnitude for the individual nano-pulse, in known giant pulse emission of several pulsars. Different emission heights for different frequencies would have to be due to propagation effects (refraction) in the pulsar magnetosphere. A detailed appraisal of these issues is in preparation.

To achieve final reliable proof of these findings, it is important to obtain even higher radio frequency data of sufficient sensitivity. Future observations of single pulses at very high frequencies combined with very high sensitivity should reveal the elementary emission processes we propose here in ordinary pulsars. Our present observations extend the spectra of observed pulsars into the long $\mathrm{mm}$ wavelength range. So far only the 
detection of one object, PSR $0355+54$, has been made at $87 \mathrm{GHz}$ with the Pico Veleta 30-m telescope (Morris et al. 1997) at the $S=0.5 \pm 0.2 \mathrm{mJy}$ level. Observations of nine pulsars at $\lambda=0.87 \mathrm{~mm}(345 \mathrm{GHz})$ have not been successful (Löhmer et al. 2004). The advent of ALMA should change this. ALMA (www. eso.org/projects/alma/) will have observational capabilities in ten frequency bands. Initially the frequency bands 84-116 GHz, 275-373 GHz, and $602-720 \mathrm{GHz}$ will be available. An IF bandwidth (in 2 polarisations) of $8 \mathrm{GHz}$ will be provided. The flux sensitivity of ALMA is expected to be sub-mJy (about $0.1 \mathrm{mJy}$ ) range after $10 \mathrm{~min}$ of integration. Considering a 4-h integration, a detection level of roughly $0.05 \mathrm{mJy}$ should be reached. This would allow the study of the flux densities of numerous pulsars even if they do not show a turn-up in their spectra. By these observations the gap between radio and optical (IR) flux detections could be closed, giving us new information about the emission mechanism inherent to pulsars. For a proper assessment of the range of coherent emission from pulsars one needs more information about the spectral behaviour of pulsars at frequencies below $100 \mathrm{MHz}$ where observational data is comparatively rare. Here pulsars are usually fairly strong radio sources with flux densities in the range of several Jy. The new generation of large low-frequency interferometers (LOFAR and possibly LWA) will be able to make detailed spectral measurements over several octaves on metre and dekametric wavelengths (Eilek et al. 2006; Stappers et al. 2006). The knowledge about the upper and lower limits of coherent pulsar radio emission is expected to become a decisive constraint on pulsar radio emission theories.

Acknowledgements. The authors would like to thank the staff of the radioobservatory Effelsberg for their help and support during the observations. We would also like to thank Harald Lesch, Jan Kuijpers and Francis Graham-Smith for encouraging discussions about the shape of pulsar spectra. We are grateful to the anonymous referee for helpful advice.

\section{References}

Bess, L. 1953, Phys. Rev., 91, 1569

Camilo, F., Ransom, S. M., Penalver, J., et al. 2007, ApJ, in press, [arXiv:0705.4095]

Conway, R. G., Kellermann, K. I., \& Long, R. J. 1963, MNRAS, 125, 261

Crusius-Waetzel, A. R., \& Lesch, H. 2002, in WE-Heraeus Seminar on Neutron Stars, Pulsars, and Supernova Remnants, ed. W. Becker, H. Lesch, \& J. Trümper, Max-Plank-Institut für Extraterrestrische Physik, Garching, 162

Eilek, J. A., Hankins, T. H. 2006, in 363rd-Heraeus Seminar on Neutron Stars and Pulsars, in press

Eilek, J. A., Hankins, T. H., \& Jessner, A. 2004, Pulsar Physics at Low Frequencies, in From Clark Lake to the Long Wavelength Array: Bill Erickson's Radio Science, Proc. Conf. held 8-11 September, in Santa Fe, New Mexico, ed. N. Kassim, M. Perez, M. Junor, \& P. Henning, USA, ASP Conf. Ser., 345, 499
Gurevich, A., Beskin, V., \& Istomin, Y. 1993, in Physics of the Pulsar Magnetosphere, ed. A. Gurevich, V. Beskin, \& Y. Istomin, ISBN 0521417465 (Cambridge, UK: Cambridge University Press), 432

Hankins, T. H., Kern, J. S., Weatherall, J. C., \& Eilek, J. A. 2003, Nature, 422, 141

Jaroschek, C. H., Lesch, H., Jessner, A. 2006, in 363rd-Heraeus Seminar on Neutron Stars and Pulsars, in press

Jenet, F.A., Anderson, S. B., \& Prince, T. A. 2001, ApJ, 558, 302

Jessner, A. 1996, in Large Antennas in Radio Astronomy, ed. C. G. M. van't Klooster, \& A. van Ardenne, 185

Jessner, A., Slowikowska, A., Klein, B., et al. 2005, Adv. Space Res., 35, 1166

Karastergiou, A., \& Johnston, S. 2004, MNRAS, 352, 689

Karastergiou, A., \& Johnston, S. 2006, MNRAS, 365, 353

Karastergiou, A., Johnston, S., \& Manchester, R. N. 2005, MNRAS, 359, 481

Kijak, J., \& Maron, O. 2004, in Young Neutron Stars and Their Environments, ed. F. Camilo, B. M. Gaensler, IAU Symp., 218 (San Francisco: ASP), 339

Kijak, J., Kramer, M., Wielebinski, R., \& Jessner, A. 1998, A\&AS, 127, 153

Kramer, M. 1994, A\&AS, 107, 527

Kramer, M. 1995, Ph.D. Thesis, University of Bonn

Kramer, M., Wielebinski, R., Jessner, A., Gil, J. A., \& Seiradakis, J. H. 1994, A\&AS, 107, 515

Kramer, M., Xilouris, K. M., Jessner, A., Wielebinski, R., \& Timofeev, M. 1996, A\&A, 306, 867

Kramer, M., Jessner, A., Doroshenko, O., \& Wielebinski, R. 1997, ApJ, 489, 364

Kramer, M., Karastergiou, A., Gupta, Y., et al. 2003, A\&A, 407, 655

Kramer, M., Lyne, A. G., O’Brien, J. T., Jordan, C. A., \& Lorimer, D. R. 2006, Science, 312, 549

Kramer, M., Stappers, B. W., Jessner, A., Lyne, A. G., \& Jordan, C. A. 2007, MNRAS, 377,107

Löhmer, O., Wolszczan, A., \& Wielebinski, R. 2004, A\&A, 425, 763

Lorimer, D. R., \& Kramer, M. 2005, Handbook of Pulsar Astronomy (Cambridge University Press)

Malofeev, V. M., Gil, J. A., Jessner, A., et al. 1994, A\&A, 285, 201

Manchester, R. N., \& Taylor, J. H. 1977, Pulsars (San Francisco: Freeman)

Maron, O., Kijak, J., Kramer, M., \& Wielebinski, R. 2000b, A\&AS, 147, 195

Melrose, D., Miller, A., Karastergiou, A., \& Luo, Q. 2006, MNRAS, 365, 638

Michel, F. C. 1991, Theory of Neutron Star Magnetospheres (Chicago: University of Chicago Press)

Moffett, D. A., \& Hankins, T. H. 1996, ApJ, 468, 779

Moffett, D. A., \& Hankins, T. H. 1999, ApJ, 522, 1046

Morris, D., Kramer, M., Thum, C., et al. 1997, A\&A, 322, L17

Ochelkov, Yu.P., \& Usov, V.V. 1984, Nature, 309, 332

Peng, B., Kraus, A., Krichbaum, T. P., \& Witzel, A. 2000, A\&AS, 145, 1

Petrova, S. A. 2001, A\&A, 378, 883

Pfeiffer H. 1959, Elektronisches Rauschen Bd. (Leipzig: Teubner) 1, 50

Romani, R. W., \& Yadigaroglu, I.-A. 1995, ApJ, 438, 314

Sieber, W. 1973, A\&A, 28, 237

Sieber, W., \& Wielebinski, R. 1987, A\&A, 177, 342

Smith, F. G. 1977, Pulsars (Cambridge University Press)

Stappers, B. 2006, in 363rd-Heraeus Seminar on Neutron Stars and Pulsars, in press

von Hoensbroech A., \& Lesch H. 1999, A\&A, 342, L57

Weatherall, J. C. 1998, ApJ, 506, 341

Weltevrede, P., Edwards, R. T., \& Stappers, B. W. 2006, A\&A, 445, 243

Wielebinski, R., Jessner, A., Kramer, M., \& Gil, J. A. 1993, A\&A, 272, L13

Xilouris, K. M., Kramer, M., Jessner, A., Wielebinski, R., \& Timofeev, M., 1996, A\&A, 309, 481 